\title{
Clinical outcomes and survival analysis of Remdesivir as a treatment option for moderate to severe COVID-19 patients.
}

Iman Aboelsaad

Egypt Ministry of Health and Population

Rasha Ashmawy ( $\square$ mri.rasha.m.informatics19@alexu.edu.eg)

Egypt Ministry of Health and Population https://orcid.org/0000-0003-2689-5968

Doaa Mahrous

Egypt Ministry of Health and Population

Sandy Sharaf

Egypt Ministry of Health and Population

Shahinda Aly

Egypt Ministry of Health and Population

Sara Abdullatif

Egypt Ministry of Health and Population

Ayat Fakhry

Egypt Ministry of Health and Population

Basma Hassan

Egypt Ministry of Health and Population

Dalia Khamis

Egypt Ministry of Health and Population

Alaa Aldakhs

Egypt Ministry of Health and Population

Ehab Kamal

NRC: National Research Center Inc

\section{Research Article}

Keywords: COVID-19, Remdesivir, antiviral treatment, and COVID-19 treatment

Posted Date: September 2nd, 2021

DOI: https://doi.org/10.21203/rs.3.rs-848909/v1

License: (c) (1) This work is licensed under a Creative Commons Attribution 4.0 International License. Read Full License 


\section{Abstract}

Background: Remdesivir is a broad-spectrum antiviral approved as promising medicine worldwide for deadly pandemic COVID-19 disease. The debate of its efficacy is interesting between different studies with consideration of several factors. We planned this study to evaluate a huge clinical outcome (primary composite outcome) of mortality rate, need for MV, and escalation of care among Remdesivir (RDV) and non-Remdesivir (NoRDV)groups.

Methods: Patients with a PCR - confirmed diagnosis of moderate and severe COVID-19 were observed retrospectively as two comparative groups, before and after including remdesivir in the treatment protocol, from August 2020 to February 2021.

Result: From 509 hospitalized patients; $35 \%$ received Remdesivir among them $64 \%$ were severe patients. Median age, 59 years, was equal in both groups, and there was no significant difference between the two groups regarding gender, baseline characteristics, and co-morbidities. Unlike, the median hospital length of stay was lower among the RDV group (8 days) than the NoRDV (9 days), $\mathrm{P}=0.004$.

The composite outcome occurred in $17.7 \%$ in RDV and $22.2 \%$ in NoRDV but the difference was statistically insignificant (p-value 0.289 ). Adjusted logistic regression showed a non-significant lower association of the composite outcome with RDV use (OR 0.623, 95Cl\% 0.37-1.02), and a significant reduction occurred in patients $<60$ years old $(\mathrm{OR} 0.39,95 \% \mathrm{Cl} 0.17-0.83)$. However, survival analysis for mortality, MV, and transfer to a higher level revealed insignificant differences in the median time between groups. Subgroup analyses showed that RDV utilization had a non-significant effect on the risk of all three outcomes across different groups.

Conclusion: Despite controlling all patient characteristics, treatment with RDV did not show any improved impact on patient outcomes over other antivirals and standard care. There is a pressing need for further studies to explore and evaluate new therapeutic approaches or combinations.

\section{Introduction}

Coronavirus disease 2019 (COVID-19) is an ongoing global deadly pandemic that emerged first in Wuhan, China in December 2019 and spread to the rest of the world. On January $30^{\text {th }}$, the world health organization declared it as a" public health emergency of international concern". The disease had serious manifestations ranging from pneumonia to death passing by multiple organ failure. Today confirmed cases approximately 177 million with over 3.84 million deaths (1). With no evidence of effective treatment, drastic non-therapeutic measures were applied worldwide to control the spread of the infection including cordon sanitaire, travel restrictions, partial or even complete lockdown (2). Eighteen months later, the situation is extremely disparate; some countries have reached zero new cases, some reached a plateau and others are still struggling to reduce infection and mortality rates with the rapid evolvement of multiple lethal variants of the virus and the vaccine distribution inequalities.

Since the very beginning, the pandemic has prompted research centers worldwide to explore the effectiveness of every existing drug or to develop novel treatments during this unprecedented public health emergency (3). The severe acute respiratory syndrome coronavirus (SARS-CoV-2) pertains to the Beta coronavirus family that comprises SARS-CoV and Middle East respiratory syndrome CoV (MERS-CoV). Consequently, every drug showing some effectiveness in patients with MERS or SARS in addition to broad-spectrum antivirals has been investigated in COVID-19 (4).

Remdesivir (RDV) is a broad-spectrum antiviral developed, in 2009, initially to treat hepatitis C (HCV) and respiratory syncytial virus (RSV) but showed no marked effect. It was then repurposed in 2015 and tested on Ebola virus disease; results confirmed its safety profile but it showed significantly lower effectiveness than monoclonal antibody treatment (5). It is a prodrug administered intravenously and activated intracellularly into the active form - a ribonucleotide analog which inhibits the viral DNA-dependent RNA polymerase activity decreasing the production of viral RNA and hence hindering its replication (6).

During the COVID-19 pandemic, in March 2020, the United States president announced the availability of RDV for "compassionate use". And due to the absence of a definitive cure, RDV was granted approval or authorization for emergency use, in approximately 50 countries, to treat COVID-19 (7). In May, The Food and Drug Authority (FDA) Authorized the Emergency Use of RDV in COVID-19 patients requiring hospitalization (8). The final drug approval as "the first antiviral for the treatment of Covid-19" five months later was issued based on the results of three randomized controlled trials that demonstrated its effectiveness in improving recovery and reducing the average length of hospital stay, among mild to severe COVID-19 (8-11).

In May, the Egyptian COVID-19 treatment protocol introduced RDV to be used in severe and critically ill cases (12). But after updated protocol in November and on, the use of the drug was expanded to include severe and moderate cases in high-risk populations (13).

Surprisingly, in November, the World Health Organization advised against the use of RDV in hospitalized patients after reviewing pertinent data and concluding a non-meaningful effect of RDV on mortality, length of stay, or even need of mechanical ventilation (MV) (3). The recommendation was based on the results of a living systematic review and network meta-analysis of 4 clinical trials including the worldwide Solidarity trial - A multinational study in which Egypt has participated $(14,15)$

In Europe, this controversial role of RDV has raised doubts in some national regulatory agencies about the need to keep it in the treatment protocol especially when considering its high cost (16). New evidence supporting or refuting the need for RDV is imperative to guide Egyptian regulator's decision-making. Hence, our study aims to assess the impact of introducing RDV in treatment protocol on patient outcomes.

Specific aim: To compare the mortality rate and the hospital length of stay, the need for MV before and after the introduction of RDV, in moderate to severe COVID-19 patients. We hypothesized that introducing RDV improved outcomes: reduced mortality rate, the escalation to a higher level of care, the need for MV, and lessened ICU and ward length of stay in moderate COVID-19 patients. 


\section{Methodology}

Study design: We proposed a retrospective observational comparative follow-up study comparing the mortality rates, need for MV and ICU, and ward length of stay in moderate to severe COVID-19 patients before and after including RDV in the COVID-19 treatment protocol.

Study setting: El-Mamourah chest hospital: a specialized chest hospital converted to COVID-19 isolation hospital during the pandemic with 84 beds in isolation wards and 42 critical care beds.

Study sample: All patients fulfilling inclusion criteria from the 1st of August till the end of February.

Study population: All patients admitted to hospital to general wards or intermediate care with a confirmed diagnosis of moderate and severe COVID-19, from August first until October 31st (NoRDV cohort); from November till February, confirmed cases receiving RDV will be included (RDV cohort). A confirmed COVID19 case was defined as a patient having a positive result of polymerase chain reaction (PCR) test for SARS-CoV-2 with a deep nasopharyngeal swab analyzed by the centralized molecular biology laboratory of the Alexandria Fever hospital $(12,13)$.

\section{Inclusion criteria}

18 years or older patients meeting definition of moderate to severe COVID-19. Moderate COVID-19 were patients whose chest radiography showed pulmonary infiltrates and SpO2 $\geq 92 \%$,whereas, severe COVID-19 patients were those with respiratory rate $>30$ breaths/min or lung infiltrates $>50$ and SpO2 $<92 \%$, $\mathrm{PaO} 2 / \mathrm{FiO} 2<300 .(12,13)$.

\section{Exclusion criteria}

Patients admitted to critical care, transferred from other hospitals, hospitalised for $<24$ hours or expired within 24 hours of admission, in addition to patients after November 1st who did not receive RDV due to the presence of contraindications.

Data collection: Data were collected retrospectively from the hospital's medical records at the pre-specified period and then recorded in the electronic data collection form.

Primary outcome definition: The primary endpoint is a composite outcome measure of escalation to an ICU from a general ward, progression to MV due to respiratory failure, or all-cause mortality.

Other outcomes: Mortality rate is defined as the number of deaths divided by the sum of patient days calculated monthly. The MV rate is the number of admitted patients who deteriorated and required MV per total patient days. Also, the average monthly length of stay (LOS) is calculated by summing all individual lengths of stay. LOS was assessed for patients who were discharged alive.

\section{Statistical analysis:}

Descriptive of the baseline characteristics of the remdesivir (RDV) and non-remdesivir (NoRDV) groups were presented as frequency and percent for categorical variables, median and range since for quantitative variables as were abnormally distributed. Chi-squared (or Fischer exact test or Monte Carlo simulation), or Mann Whitney were used for comparison of the two groups. mortality rates difference between groups was assessed by the Z test for independent rates. The median length of stay in the RDV and NoRDV was compared using the Mann-Whitney test.

Bivariate Logistic regression analysis was performed for the composite measure as dependent and the use of RDV as an explanatory variable, a subsequent multivariate analysis was conducted to adjust for age, gender, disease severity, and comorbidities. Variables were included in the model if the P-value of the univariate analysis was lower than 0.2 .

Survival analysis was carried out using the Log Rank test to compare median time to death, to MV, and transfer to higher level separately across groups. Multivariate Cox proportional regression analysis was then conducted to assess the effect of RDV on mortality rate, MV rate, and transfer to higher level rate while adjusting for all other predictors. A p-value of 0.05 is deemed statistically significant. subgroup analyses were performed for mortality and MV using the same regression model. Our sample surpassed the minimum required sample size which was 213 in both groups, calculated assuming that the proportion of composite outcome in RDV and NoRDV groups equal $35 \%$ and $54 \%$ respectively (17), to achieve $80 \%$ power at a $5 \%$ level of significance was. All statistical analyses and sample size calculations were performed using $\mathrm{R}$ software (R version 4.0.5).

\section{Timeline}

Data collection was from the first of February till the end of March.

Ethical considerations: Review and approval of the protocol were obtained by the Ministry of Health and Population (MoHP) research ethics committee (Com. No/Dec. No: 3-2021/22), with waivered informed consent due to the retrospective nature of the study.

\section{Results:}

A total of 509 hospitalized patients were included in the study (PCR-confirmed COVID -19), 175 (35\%) of which were in the RDV group and 334 (65\%) in the NoRDV group. The median and minimum age of both groups were similar $(59,25$ years), while the maximum age in the RDV cohort was 91 and for the NoRDV cohort 89 years old. Females represent $55.4 \%$ of the NoRDV group, $46.9 \%$ of the RDV group; Alexandria residents constituted $88.9 \%$ and $89.7 \%$ of the RDV and 
the non-RDV group, respectively, from which 197 patients (40.1\% NoRDV, 36\% RDV group) were categorized as having moderate disease, and 312 (59.9\% NoRDV, 64\% RDV group) were in the severe disease stratum. There is no statistical difference in the distribution of disease severity between the groups, $\mathrm{P}=0.418$. The baseline characteristics were well balanced between the groups concerning biochemical laboratory tests (SGOT, SGPT, urea, creatinine, leucocytes cells $/ \mathrm{mm}$, lymphocytes\%, vital signs (temperature, heart rate, respiratory rate, oxygen saturation, diastolic and systolic blood pressure), and the comorbidities as shown in (Table 1).

Each patient included in the study received different therapeutic management according to the continued updates in COVID protocol according to MoHP Egypt. 
Table 1: Baseline patient characteristics and clinical manifestations.

\begin{tabular}{|c|c|c|c|c|c|c|c|c|c|c|}
\hline & \multirow{2}{*}{$\begin{array}{l}\text { characteristics } \\
\text { Remdesivir use }\end{array}$} & \multicolumn{3}{|l|}{ Overall } & \multicolumn{2}{|l|}{ Moderate } & \multicolumn{4}{|c|}{ Severe } \\
\hline & & $\begin{array}{l}\text { No } \\
(\mathrm{N}=334)\end{array}$ & $\begin{array}{l}\text { Yes } \\
(\mathrm{N}=175)\end{array}$ & $\begin{array}{l}\mathrm{P} \text { - } \\
\text { value }\end{array}$ & $\begin{array}{l}\text { No } \\
(\mathrm{N}=134)\end{array}$ & $\begin{array}{l}\text { Yes } \\
(\mathrm{N}=63)\end{array}$ & $\begin{array}{l}\mathrm{P}- \\
\text { value }\end{array}$ & $\begin{array}{l}\text { No } \\
(\mathrm{N}=200)\end{array}$ & $\begin{array}{l}\text { Yes } \\
(\mathrm{N}=112)\end{array}$ & $\begin{array}{l}\mathrm{P}- \\
\text { value }\end{array}$ \\
\hline \multirow[t]{4}{*}{ Demographics } & Female Gender & $\begin{array}{l}185 \\
(55.4 \%)\end{array}$ & $\begin{array}{l}82 \\
(46.9 \%)\end{array}$ & 0.0823 & $71(53 \%)$ & $\begin{array}{l}25 \\
(39.7 \%)\end{array}$ & 0.112 & $\begin{array}{l}114 \\
(57.0 \%)\end{array}$ & $57(50.9 \%)$ & 0.357 \\
\hline & Age (years) & 59 & 59 & 0.864 & 59 & 60 & 0.905 & 58.5 & 59 & 0.718 \\
\hline & & {$[25,91]$} & {$[25,89]$} & & {$[25,91]$} & {$[27,84]$} & & {$[27,88]$} & {$[25,89]$} & \\
\hline & Alexandria residents & $\begin{array}{l}297 \\
(88.9 \%)\end{array}$ & $\begin{array}{l}157 \\
(89.7 \%)\end{array}$ & 0.902 & $\begin{array}{l}119 \\
(88.8 \%)\end{array}$ & $61(96.8 \%)$ & 0.0994 & $\begin{array}{l}178 \\
(89 \%)\end{array}$ & $\begin{array}{l}96.0 \\
(85.7 \%)\end{array}$ & 0.502 \\
\hline \multirow[t]{13}{*}{ Vital signs } & Disturbed consciousness & $\begin{array}{l}31 \\
(9.3 \%)\end{array}$ & $13(7.4 \%)$ & 0.589 & $2(1.5 \%)$ & $0(0 \%)$ & 1 & $\begin{array}{l}29 \\
(14.5 \%)\end{array}$ & $\begin{array}{l}13 \\
(11.6 \%)\end{array}$ & 0.586 \\
\hline & Temperature $\left({ }^{\circ} \mathrm{C}\right)$ & 37 & 37 & 0.0083 & 37 & 37 & 0.461 & 37 & 37 & 0.0102 \\
\hline & & {$[35,39]$} & {$[36,39]$} & & {$[36,39]$} & {$[36.2,39]$} & & {$[35,39]$} & {$[36,39]$} & \\
\hline & Heart Rate & 86 & 85 & 0.211 & 85.5 & 85 & 0.281 & 86 & 86 & 0.406 \\
\hline & & $\begin{array}{l}{[22,} \\
118]\end{array}$ & {$[30,103]$} & & $\begin{array}{l}{[22,} \\
118]\end{array}$ & {$[30,103]$} & & {$[22,118]$} & {$[52,101]$} & \\
\hline & Respiratory Rate & 22 & 22 & $<0.001$ & 22 & 22 & $<0.001$ & 22 & 22.0 & $<0.001$ \\
\hline & & {$[18,50]$} & {$[18,40]$} & & {$[18,30]$} & {$[18,30]$} & & {$[20,50]$} & {$[18,40]$} & \\
\hline & Oxygen saturation (\%) & 90 & 88 & 0.0338 & 95 & 95 & 0.218 & 86 & 85 & 0.0368 \\
\hline & & {$[50,99]$} & {$[43,100]$} & & {$[92,99]$} & {$[92,100]$} & & {$[50,98]$} & {$[43,91]$} & \\
\hline & Diastolic blood pressure & 120 & 120 & 0.516 & 120 & 120 & 0.854 & 120 & 120 & 0.441 \\
\hline & & {$[90,180]$} & $\begin{array}{l}{[100,} \\
160]\end{array}$ & & $\begin{array}{l}{[100,} \\
160]\end{array}$ & $\begin{array}{l}\text { [100, } \\
150]\end{array}$ & & {$[90,180]$} & {$[110,160]$} & \\
\hline & Systolic blood pressure & 80 & 80 & 0.623 & 80 & 80 & 0.251 & 80 & 80 & 0.84 \\
\hline & & {$[20,100]$} & {$[50,100]$} & & {$[60,95]$} & {$[50,95]$} & & $\begin{array}{l}{[20} \\
100]\end{array}$ & {$[70,100]$} & \\
\hline \multirow{12}{*}{$\begin{array}{l}\text { Laboratory } \\
\text { investigations }\end{array}$} & SGOT (U/L) & 31 & 30.5 & 0.98 & 31 & 32 & 0.93 & 30 & 30 & 0.952 \\
\hline & & {$[10,400]$} & {$[8,225]$} & & {$[10,400]$} & {$[8,137]$} & & $\begin{array}{l}{[10} \\
362]\end{array}$ & {$[10,225]$} & \\
\hline & SGPT (U/L) & 29 & 28 & 0.642 & 29 & 29 & 0.868 & 30 & 26 & 0.668 \\
\hline & & {$[7,419]$} & {$[9,192]$} & & {$[8,419]$} & {$[11,72]$} & & {$[7,203]$} & {$[9,192]$} & \\
\hline & Urea (mg/dl) & 39 & 39 & 0.683 & 35 & 41 & 0.233 & 40 & 38.5 & 0.556 \\
\hline & & $\begin{array}{l}{[0.38,} \\
320]\end{array}$ & {$[1,215]$} & & $\begin{array}{l}{[0.38} \\
32]\end{array}$ & {$[15,215]$} & & $\begin{array}{l}{[12,} \\
148]\end{array}$ & {$[1,136]$} & \\
\hline & Creatinine $(\mathrm{mg} / \mathrm{dl})$ & 1 & 1 & 0.118 & 0.9 & 1 & 0.617 & 1 & 1.1 & 0.151 \\
\hline & & {$[0.5,5.6]$} & {$[0.4,3.9]$} & & {$[0.5,5.6]$} & {$[0.4,3.7]$} & & {$[0.5,3.1]$} & {$[0.4,3.9]$} & \\
\hline & Leucocytes (cells $/ \mathrm{mm}^{3} * 10^{3}$ ) & 8.3 & 8.5 & 0.752 & 7.5 & 7.75 & 0.754 & 8.5 & 9.7 & 0.826 \\
\hline & & {$[1.8,71]$} & $\begin{array}{l}{[1.88,} \\
32.6]\end{array}$ & & $\begin{array}{l}{[2.3} \\
20.8]\end{array}$ & $\begin{array}{l}{[1.88,} \\
32.6]\end{array}$ & & {$[1.8,71]$} & {$[3.6,31.1]$} & \\
\hline & Lymphocytes\% & 15.5 & 16 & 0.326 & 17 & 22 & 0.146 & 15 & 14 & 0.829 \\
\hline & & {$[2,91]$} & {$[4,90]$} & & {$[3,90]$} & {$[4,90]$} & & {$[2,91]$} & {$[6,86]$} & \\
\hline \multirow{7}{*}{$\begin{array}{l}\text { Coexisting } \\
\text { disease }\end{array}$} & Sarcoidosis & $1(0.3 \%)$ & $0(0 \%)$ & 1 & $0(0 \%)$ & $0(0 \%)$ & 1 & $1(0.5 \%)$ & $0(0 \%)$ & 1 \\
\hline & Obesity & $1(0.3 \%)$ & $3(1.7 \%)$ & 0.12 & $0(0 \%)$ & $1(1.6 \%)$ & 0.32 & $1(0.5 \%)$ & $2(1.8 \%)$ & 0.293 \\
\hline & Diabetes & $\begin{array}{l}160 \\
(47.9 \%)\end{array}$ & $79(45.1 \%)$ & 0.617 & $\begin{array}{l}56 \\
(41.8 \%)\end{array}$ & $27(42.9 \%)$ & 1 & $\begin{array}{l}104 \\
(52.0 \%)\end{array}$ & $52(46.4 \%)$ & 0.409 \\
\hline & COPD & $3(0.9 \%)$ & $0(0 \%)$ & 0.555 & $1(0.7 \%)$ & $0(0 \%)$ & 1 & $2(1.0 \%)$ & $0(0 \%)$ & 0.538 \\
\hline & Chronic liver disease & $3(0.9 \%)$ & $1(0.6 \%)$ & 1 & $1(0.7 \%)$ & $1(1.6 \%)$ & 0.538 & $2(1.0 \%)$ & $0(0 \%)$ & 0.538 \\
\hline & History DVT & $2(0.6 \%)$ & $0(0 \%)$ & 0.548 & $1(0.7 \%)$ & $0(0 \%)$ & 1 & $1(0.5 \%)$ & $0(0 \%)$ & 1 \\
\hline & ILD & $2(0.6 \%)$ & $2(1.1 \%)$ & 0.61 & $1(0.7 \%)$ & $1(1.6 \%)$ & 0.538 & $1(0.5 \%)$ & $1(0.9 \%)$ & 1 \\
\hline
\end{tabular}




\begin{tabular}{llllllllll} 
hypothyroid & $6(1.8 \%)$ & $1(0.6 \%)$ & 0.431 & $1(0.7 \%)$ & $0(0 \%)$ & 1 & $5(2.5 \%)$ & $1(0.9 \%)$ & 0.425 \\
Pregnancy & $0(0 \%)$ & $1(0.6 \%)$ & 0.344 & $0(0 \%)$ & $1(1.6 \%)$ & 0.32 & $0(0 \%)$ & $0(0 \%)$ & 1 \\
\hline Old TB & $0(0 \%)$ & $1(0.6 \%)$ & 0.344 & $0(0 \%)$ & $0(0 \%)$ & 1 & $0(0 \%)$ & $\begin{array}{l}1.00 \\
(0.9 \%)\end{array}$ & 0.359 \\
\hline CKD & $6(1.8 \%)$ & $10(5.7 \%)$ & 0.0286 & $2(1.5 \%)$ & $3(4.8 \%)$ & 0.33 & $4(2 \%)$ & $7(6.3 \%)$ & 0.0608 \\
\hline Stroke & $2(0.6 \%)$ & $1(0.6 \%)$ & 1 & $0(0 \%)$ & $0(0 \%)$ & 1 & $2(1.0 \%)$ & $1(0.9 \%)$ & 1 \\
\hline Cancer & $2(0.6 \%)$ & $0(0 \%)$ & 0.548 & $1(0.7 \%)$ & $0(0 \%)$ & 1 & $1(0.5 \%)$ & $0(0 \%)$ & 1
\end{tabular}

\section{Disease Severity}

$\begin{array}{llll}\text { Moderate } & 134 & 63(36 \%) & \\ & (40.1 \%) & & \\ \text { Severe } & 200 & 112(64 \%) & 0.418 \\ & (59.9 \%) & & \end{array}$

*Data are expressed as median [min, max] for quantitative, number (percentage) for qualitative.

Test of significance used: Mann Whitney, Chi-Square, Fisher exact, Monte Carlo.

SGPT: Serum glutamic pyruvic transaminase, SGOT: Serum glutamic oxaloacetic transaminase, COPD: Chronic obstructive pulmonary disorder, ILD: Interstitial lung disease, DVT: Deep vein thrombosis, TB: Tuberculosis, CKD: Chronic lung disease.

Our total population was divided into two strata: moderate $(\mathrm{N}=197)$ and severe $(\mathrm{N}=312)$. In the moderate group, 63 (32\%) patients received RDV, and 78 (39.6\%), 6 (3\%), and 2 (1\%) received Hydroxychloroquine, Ivermectin, Lopinavir/ Ritonavir, respectively. While 112 (35.9\%) of the severe group received RDV, 74 (23.7\%), 25 (8\%), 9 (2.9\%) received Hydroxychloroquine, Ivermectin, and Lopinavir/ Ritonavir, respectively.

Outcomes: The outcomes of cohorts are presented in Table 2; patients in the RDV group had statistically significant shorter hospital length of stay (median= 8 [range 1-29] days) than patients in the NoRDV group (median $=9$ [ range 1-65] days) $(P=0.004)$, and the difference was more obvious in severe cases. 
Table 2: Patients' outcomes

\begin{tabular}{|c|c|c|c|c|c|c|c|c|c|}
\hline \multirow{2}{*}{ Remdesivir use } & \multicolumn{3}{|l|}{ Overall } & \multicolumn{2}{|l|}{ Moderate } & \multicolumn{2}{|c|}{ Severe } & \multirow[b]{2}{*}{$\begin{array}{l}\text { yes } \\
(\mathrm{N}=112)\end{array}$} & \multirow[b]{2}{*}{ P-value } \\
\hline & $\begin{array}{l}\text { No } \\
(\mathrm{N}=334)\end{array}$ & $\begin{array}{l}\text { yes } \\
(\mathrm{N}=175)\end{array}$ & P-value & $\begin{array}{l}\text { No } \\
(\mathrm{N}=134)\end{array}$ & $\begin{array}{l}\text { yes } \\
(\mathrm{N}=63)\end{array}$ & P-value & $\begin{array}{l}\text { No } \\
(\mathrm{N}=200)\end{array}$ & & \\
\hline \multicolumn{10}{|l|}{ Discharge state } \\
\hline Improved & $254(76.0 \%)$ & $134(76.6 \%)$ & 0.594 & $115(85.8 \%)$ & $54(85.7 \%)$ & 0.652 & 139 (69.5\%) & $80(71.4 \%)$ & 0.754 \\
\hline DAMA & $19(5.7 \%)$ & 12.(6.9\%) & & $5(3.7 \%)$ & $4(6.3 \%)$ & & $14(7 \%)$ & $8(7.1 \%)$ & \\
\hline Transferred & $4(1.2 \%)$ & $0(0 \%)$ & & $2(1.5 \%)$ & $0(0 \%)$ & & 2.(1\%) & $0(0 \%)$ & \\
\hline Died & $57(17.1 \%)$ & $29(16.6 \%)$ & & $12(9.7 \%)$ & $5(7.9 \%)$ & & $45(22.5 \%)$ & $24(21.4 \%)$ & \\
\hline \multirow[t]{2}{*}{ Length of stay } & 9 & 8 & 0.0042 & 9 & 8 & 0.157 & 10 & 8 & 0.0107 \\
\hline & {$[1,65]$} & {$[1,29]$} & & {$[1,46]$} & {$[1,19]$} & & {$[3,65]$} & {$[2,29]$} & \\
\hline Composite outcome & $74(22.2 \%)$ & $31(17.7 \%)$ & 0.289 & $17(12.7 \%)$ & $6(9.5 \%)$ & 0.684 & $57(28.5 \%)$ & $25(22.3 \%)$ & 0.291 \\
\hline In-hospital death & $57(17.1 \%)$ & $29(16.6 \%)$ & 10.856 & $12(9.0 \%)$ & $5(7.9 \%)$ & 1 & 45 (22.5\%) & $24(21.4 \%)$ & 0.939 \\
\hline \multirow[t]{2}{*}{ Time to death } & 10 & 8 & 0.819 & 6.5 & 8 & 0.708 & 10 & 8 & 0.96 \\
\hline & {$[4,30]$} & {$[3,27]$} & & {$[4,19]$} & {$[5,14]$} & & {$[4,30]$} & {$[3,27]$} & \\
\hline Mechanical Ventilation & $34(10.2 \%)$ & $15(8.6 \%)$ & 0.67 & $6(4.5 \%)$ & $3(4.8 \%)$ & 1 & $28(14.0 \%)$ & $12(10.7 \%)$ & 0.512 \\
\hline \multirow[t]{2}{*}{ Time to MV } & 7 & 7 & 0.965 & 5.5 & 5 & 0.358 & 7 & 10 & 0.486 \\
\hline & {$[1,30]$} & {$[1,22]$} & & {$[3,18]$} & {$[2,5]$} & & {$[1,30]$} & {$[1,22]$} & \\
\hline Transfer to higher level & $58(17.4 \%)$ & $21(12 \%)$ & 0.145 & $12(9 \%)$ & $5(7.9 \%)$ & 1 & $46(23 \%)$ & $16(14.3 \%)$ & 0.0887 \\
\hline \multirow[t]{2}{*}{ Time to transfer } & 6 & 7 & 0.0896 & 3 & 5.00 & 0.49 & 6 & 7.5 & 0.0661 \\
\hline & {$[1,36]$} & {$[0,34]$} & & {$[1,19]$} & {$[2,9]$} & & {$[1,36]$} & {$[0,34]$} & \\
\hline \multicolumn{10}{|l|}{ New level } \\
\hline ICU 1 & $2(0.6 \%)$ & $2(1.1 \%)$ & 0.188 & $0(0 \%)$ & $0(0 \%)$ & NA & $2(1 \%)$ & $2(1.8 \%)$ & 0.122 \\
\hline ICU 2 & $53(15.9 \%)$ & $18(10.3 \%)$ & & $12(9 \%)$ & $5(7.9 \%)$ & & $41(20.5 \%)$ & $13(11.6 \%)$ & \\
\hline \multirow[t]{2}{*}{ Mortality rate } & 15.9 & 17.67 & 0.6587 & 9.12 & 9.17 & 0.992 & 19.92 & 21.89 & 0.7125 \\
\hline & [11.8-2] & [11.2-24] & & [3.9-14.3] & {$[1.13-17.26]$} & & {$[14.1-25.4]$} & {$[13.1-30.6]$} & \\
\hline \multirow[t]{2}{*}{ Mechanical ventilation rate } & 9.85 & 9.37 & 0.8707 & 4.63 & 5.58 & 0.7998 & 12.98 & 11.28 & 0.6772 \\
\hline & [6.54-13.16] & {$[4.63-14.11]$} & & [0.9-8.33] & {$[-0.7-11.89]$} & & [8.18-17.79] & {$[4.9-17.67]$} & \\
\hline \multirow[t]{2}{*}{ Transfer rate } & 18.46 & 13.39 & 0.1812 & 9.13 & 9.49 & 0.9422 & 23.84 & 15.36 & 0.1032 \\
\hline & {$[13.7-23.2]$} & {$[7.66-19.11]$} & & [3.96-14.29] & {$[1.17-17.8]$} & & [16.95-30.73] & [7.83-22.89] & \\
\hline
\end{tabular}

DAMA: discharge against medical advice, ICU1: intermediate care unit, ICU 2: intensive care unit, MV: mechanical ventilation

Data are expressed as median [min, max] for quantitative, number (percentage) for qualitative.

. Mortality rate (death /1000 patient-days), mechanical ventilation rate (Episode of MV /1000 patient-days), transfer rate (Episode of transfer /1000 patientdays).

Test of significance used: Mann Whitney, Chi-Square, Fisher exact, Monte Carlo and Z test for independent rates

The primary composite outcomes consisting of death, MV, and transfer to a higher level occurred in $17.7 \%$ of the RDV group and $22 \%$ of the non-RDV group, however, the effect was not statistically significant ( $p$-value 0.289 ), even after stratification into moderate and severe cases ( $p=0.684$ and 0.291 , respectively). All-cause mortality rate was slightly higher in RDV group 17.67[11.2-4]/1000 patient days than NoRDV group 15.9 [11.8-2]/1000 patient days (Table 2).

The univariate logistic regression analysis to test the effect of RDV use revealed a non-significant lower association with RDV use (OR= 0.75 , 95\% $\mathrm{Cl} 0.47$ 1.19) which remained insignificant (OR $0.623,95 \mathrm{Cl} \% 0.37-1.02)$ after adjusting for all characteristics with $P$-value $<0.2$ on separate logistic. The patient age and COVID severity are the significant factors for our primary composite outcome $(\mathrm{OR} 1.597,2.804)$ respectively. Other factors such as gender, cardiovascular disease, chronic lung diseases, diabetes, obesity even chronic kidney disease were not significantly associated with the composite outcome (Table 3 ). 


\begin{tabular}{|lll|}
\hline \multicolumn{3}{|l|}{ Table 3: multivariate logistic regression } \\
\hline Variable & $\mathrm{OR}$ & $95 \% \mathrm{Cl}$ \\
\hline Intercept & 0.120 & $0.065-0.213$ \\
\hline Remdesivir & 0.623 & $0.337-1.018$ \\
\hline Age above 60 years & 1.597 & $1.009-2.535$ \\
\hline Male Gender & 0.789 & $0.498-1.242$ \\
\hline Severe COVID & 2.804 & $1.697-4.795$ \\
\hline Cardiovascular disease & 1.464 & $0.721-2.854$ \\
\hline Chronic lung disease & 1.260 & $0.355-3.794$ \\
\hline Chronic kidney disease & 5.524 & $1.865-17.079$ \\
\hline Diabetes & 0.912 & $0.575-1.440$ \\
\hline Obesity & 4.670 & $0.522-42.258$ \\
\hline
\end{tabular}

Multivariate logistic regression models were stratified by significant variables in the previous multivariate model. Stratifying by age groups (above or under 60 years). the use of RDV was associated with a significant reduction of the composite outcome $(0 \mathrm{R} 0.39,95 \% \mathrm{Cl} 0.17-0.83)$ in patients less than 60 years, on the other hand, the association was insignificant (OR 1.16,95\% $0.61-2.17$ ) for the patients older 65 years old. we explored which of the outcomes is associated with RDV use in a subgroup of patients below 60 years old, and we detected only a significant decrease from $17.4 \%$ to $6.4 \%$ in transfer to the higher level $(\mathrm{p}=0.01912)$. Similarly, separate logistic regression analysis revealed that only transfer to higher level had a significant association (OR 0.32 , $95 \% \mathrm{Cl} 0.12-0.75)$ but not for mortality and MV (OR $0.56,95 \% \mathrm{Cl} 0.24-1.20)$ and $(\mathrm{OR} 0.59,95 \% \mathrm{Cl} 0.21-1.45)$, respectively.

In addition, upon stratification according to COVID severity, for moderate patients, the RDV was insignificantly associated (OR $0.73,95 \% \mathrm{Cl} 0.25-1.88)$ while for severe COVID patients, the RDV showed a significant association with reduced composed outcome. (OR $0.42,95 \% \mathrm{Cl} 0.17-0.96)$. However, none of the individual outcomes was significantly associated with the RDV use ( $p=0.939,0.5117,0.0886)$ for mortality, MV, and transfer to a higher level, respectively)

Log-rank test revealed no difference in median time to death, time to MV, and time to transfer to a higher level $(P=0.48,0.89,0.23$ respectively) as shown in Kaplan-Meier curves (Figure1).

Moreover, we performed a series of univariate and multivariate Cox proportional hazard regression models for each outcome constituting our composite outcome separately (mortality, mechanical ventilation, or transfer to a higher level), to include the time factor in the analysis. The use of RDV was associated with an insignificant reduction in the MV and escalation of care and an insignificant minor increase in mortality. Results are illustrated in Table 4.

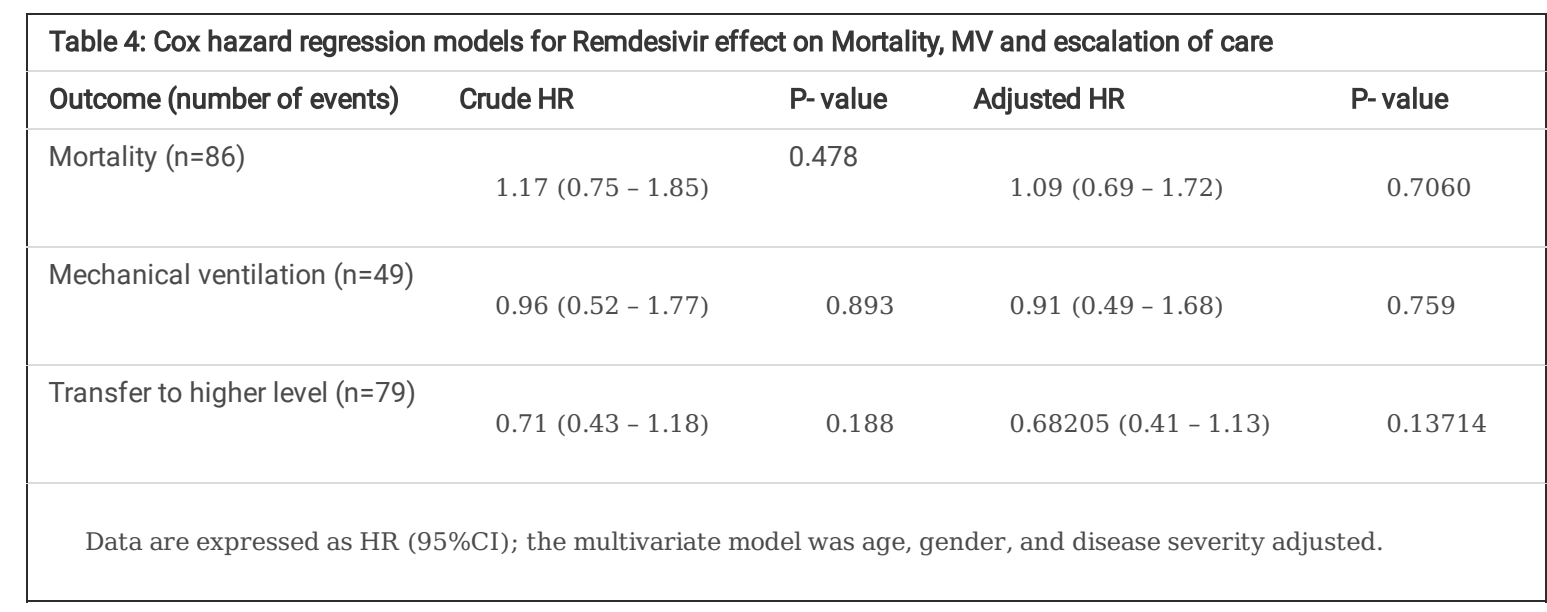

Subsequent subgroup analyses for the same model to explore effects in different subgroups revealed also a non-significant effect of RDV utilization on the risk of mortality and MV (Figure 2 and 3).

\section{Discussion:}

To the best of our knowledge, this is the first study in Egypt assessing the effectiveness of RDV compared to other regimens while encompassing all patient characteristics in real situations with limited exclusion criteria.

In this study, our primary outcome was measured as a composite outcome of death, mechanical ventilation, and transfer to a higher level of care. We could not detect a significantly different effect of RDV on the occurrence of the composite outcome (22.2\%) after its inclusion in MoHP protocol compared to those 
who received other treatments (17.7\%) in hospitalized patients with moderate to severe COVID-19. The association of the composite outcome with the use of RDV was insignificant even after adjusting for all baseline characteristics which were nearly balanced in the two cohorts. Age and disease severity were significant predictors of the composite outcome but, surprisingly, all other co-morbidities including cardiovascular diseases and lung diseases were not. This may be attributed to possible incomplete history in patient records. Moreover, in the strata of ts younger than 60 years old, the odds of patients experiencing the composite outcome are $58 \%$ lower in the RDV cohort than the NoRDV cohort with no significant effect on older patients. Nevertheless, the effect was solely detected in the transfer to a higher level with no significant reduction in mortality or need of MV.

Furthermore, no difference was detected in overall mortality rate, MV rate, and transfer to a higher level rate. A possible cause is that a comparison of RDV is conducted against a combination of antivirals and standard care, not standard care alone. The reduction in median length of stay was mainly prominent in severe COVID cases.

However, Separate adjusted Cox hazard regression models for each endpoint in our composite outcome, RDV revealed a statistically insignificant effect for all three outcomes. Crude, adjusted hazard ratios as well as subgroup analysis for mortality, MV, and transfer to a higher level are consistent with results of the systematic review and network meta-analysis conducted by Rochwerg et al. on which The WHO updated living guidelines based the advice against RDV use regardless of the disease severity $(3,15)$. The pooled data from 7333 patients revealed that RDV had no significant effect on mortality (odds ratio $0.92 ; 95 \% \mathrm{Cl}$ $, 0.80$ to 1.07$)$, the need for MV (OR $0.88 ; 95 \% \mathrm{Cl}, 0.76-1.03)$ and the duration of hospitalization (Mean Difference: -0.5 lower ; $95 \% \mathrm{Cl}, 3.3$ lower -2.3 higher) $(3$, 15). However, a recent meta-analysis pooled the results of two non-interventional studies estimated a $44 \%$ reduction in risk of 28 -days mortality (18). This discrepancy could be explained by the difference in the population since Pasquini et al. focused on critical patients on MV (19) and Fried et.al. reported the risk based on only 44 patients (20). Furthermore, a study conducted by Olender SA et al. concluded that by Day 14 RDV was associated with significantly greater recovery and $62 \%$ reduced odds of death versus standard of care treatment in severe COVID-19 patients (21). In our study, the insignificant effect may be attributed to either the difference in the comparison group since we are comparing against a combination of antivirals and standard care. Another potential explanation is lower power since our sample size was initially calculated based on the primary outcome (composite outcome) not on secondary outcomes, suggesting the need for further studies with a specifically calculated sample size to assess each outcome.

In the previously published studies supporting the use of RDV, Beigel JH et al. concluded that the RDV arm had a lower median time to recovery (10 days; $95 \%$ $\mathrm{Cl}, 9-11$ ) compared to placebo ( 15 days; $95 \% \mathrm{Cl}, 13$ to 18 ) but the effect on mortality was not statistically significant (hazard ratio $0.73 ; 95 \% \mathrm{Cl}, 0.52-1.03$ ) (10). Spinner et al, in an open-label clinical trial, revealed that $65 \%$ and $54 \%$ of patients receiving a 5 -day course of RDV and patients receiving a 10 -day course, respectively, had a 2 points clinical improvement at day 14 on a 7-point ordinal scale. This indicates a significant clinical improvement from baseline for both groups and no statistically significant benefit of the 10-day course (11). In the third study, hospitalized moderate COVID-19 patients receiving a 5-day course of RDV had a statistically significant improvement in clinical status compared to those on standard care (odds ratio $=1.65 ; 95 \% \mathrm{Cl}, 1.09-2.48$ ) after 11 days from treatment initiation. However, the improvement was of vague clinical importance (9). In our study, a similar improvement percentage was observed in the RDV group (76.6\%) and the NoRDV group (76.0\%), yet we were not able to assess quantitatively the effect of RDV on clinical improvement since no similar instrument is applied in our local hospital.

Based on our data, the treatment with RDV did not reduce the need for MV nor prevented the progression to severe respiratory distress or even death when compared to other regimens.

RDV was the promising medicine for COVID-19 after "Food and Drug Administration (FDA) issued an Emergency Use Authorisation to permit the use of RDV for treatment of COVID-19 patients" based on findings of different studies showing an encouraging effect for the use of RDV in COVID-19 (9-11, 22). Later, on November 20, 2020, WHO issued a conditional recommendation against the use of RDV after disappointing results from the SOLIDARITY trial and the absence of concrete evidence from other studies that the survival outcomes were improved by RDV. Consequently, WHO excluded RDV from the COVID-19 management list with no further updates (3). Our results coincide with these recommendations. Accordingly, we urge the Egyptian MoHP to widely explore the effect of RDV in other settings and conduct cost-effectiveness analyses to evaluate RDV before the next COVID-19 patient management Protocol revision.

Limitations:

This study is a one-center study, so our results could only be applied to a population similar to ours. Additionally, the retrospective comparative before and after the design is not the best design to assess effectiveness, but due to the availability of records before and after the guidelines' application, the time limitation, the inability of the research team to randomize the treatment, and the absence of a comparable control group in the same period, this design was considered the optimal. Also, the retrospective nature of the study, data were collected from a single source - the paper-based patient medical record - so several important variables were missing and could not be ascertained otherwise.

\section{Conclusion:}

Our data show that treatment with RDV did not show an improved impact on patient outcomes over other antivirals and standard care, including the need for $\mathrm{MV}$, the progression of the disease to severe respiratory distress, or even death. There is a pressing need for further studies to explore and evaluate new therapeutic approaches: novel antivirals, immuno-modulators, and combination therapy, to improve outcomes in patients with COVID-19 and effectively aid the fight against this menacing pandemic.

\section{Declarations}

\section{Conflict of interest:}


All authors declared no conflict of interest.

\section{Funding:}

No funding

\section{Author contributions:}

- Conceptualization: [Iman Aboelsaad, Rasha Ashmawy, Alaa Aldakhs, Ehab Kamal, Basma Hassan]; Methodology: [Iman Aboelsaad, Rasha Ashmawy]; Material preparation, data collection, data preparation: [Basma Hassan, Ayat Fakhry, Shahinda Aly, Doaa Mahrous, Sandy Sharaf, Sara Abdullatif, Rasha Ashmawy, Dalia Khamis]; Data Analysis [Iman Aboelsaad]; Writing - original draft preparation: [Iman Aboelsaad, Rasha Ashmawy, Doaa Mahrous, Sandy Sharaf ]; Writing - review and editing: [Basma Hassan, Ayat Fakhry, Shahinda Aly, Sara Abdullatif, Dalia Khamis]; Supervision: [Alaa Aldakhs, Ehab Kamal ]

\section{References}

1. World Health Organization. WHO Coronavirus Disease (COVID-19) Dashboard 2020. Available from: https://covid19.who.int/.

2. Dryhurst S, Schneider CR, Kerr J, et al. Risk perceptions of COVID-19 around the world. Journal of Risk Research. 2020:1-13. doi:10.1080/13669877.2020.1758193.

3. World Health Organization. Therapeutics and COVID-19: living guideline, 20 November 2020. Geneva: World Health Organization, 2020 2020. Report No.: Contract No.: WHO/2019-nCov/remdesivir/2020.1.

4. Eastman RT, Roth JS, Brimacombe KR, et al. Remdesivir: A Review of Its Discovery and Development Leading to Emergency Use Authorization for Treatment of COVID-19. ACS Central Science. 2020;6(5):672-83. doi:10.1021/acscentsci.0c00489.

5. Agostini ML, Andres EL, Sims AC, et al. Coronavirus Susceptibility to the Antiviral Remdesivir (GS-5734) Is Mediated by the Viral Polymerase and the Proofreading Exoribonuclease. mBio. 2018;9(2):e00221-18. doi:10.1128/mBio.00221-18.

6. Ferner RE, Aronson JK. Remdesivir in covid-19. BMJ. 2020;369:m1610. doi:10.1136/bmj.m1610.

7. U.S. Food and Drug Administration Approves Gilead's Antiviral Veklury® (remdesivir) for Treatment of COVID-19. 2020. https://www.gilead.com/newsand-press/press-room/press-releases/2020/10/us-food-and-drug-administration-approves-gileads-antiviral-veklury-remdesivir-for-treatment-of-covid19.

8. Rubin D, Chan-Tack K, Farley J, et al. FDA Approval of Remdesivir - A Step in the Right Direction. N Engl J Med. 2020. doi:10.1056/NEJMp2032369.

9. Goldman JD, Lye DCB, Hui DS, et al. Remdesivir for 5 or 10 Days in Patients with Severe Covid-19. N Engl J Med. 2020;383(19):1827-37. doi:10.1056/NEJMoa2015301.

10. Beigel JH, Tomashek KM, Dodd LE, et al. Remdesivir for the Treatment of Covid-19 - Final Report. N Engl J Med. 2020;383(19):1813-26. doi:10.1056/NEJMoa2007764.

11. Spinner CD, Gottlieb RL, Criner GJ, et al. Effect of Remdesivir vs Standard Care on Clinical Status at 11 Days in Patients With Moderate CoVID-19: A Randomized Clinical Trial. JAMA. 2020;324(11):1048-57. doi:10.1001/jama.2020.16349.

12. Management protocol for COVID-19 patients. Egyptian Ministry of Health. Version 1.4 /30th May 2020.. http://www.mohp.gov.eg/JobsDetails.aspx? job_id=3061.

13. Management protocol for COVID-19 patients. Ministry of health and population, Egypt. version 1.4 (November 2020). http://www.mohp.gov.eg/JobsDetails.aspx?job_id=3061.

14. Pan H, Peto R, Henao-Restrepo AM, et al. Repurposed Antiviral Drugs for Covid-19 - Interim WHO Solidarity Trial Results. N Engl J Med. 2021;384(6):497511. doi:10.1056/NEJMoa2023184.

15. Rochwerg B, Agoritsas T, Lamontagne F, et al. RAPID RECOMMENDATIONS A living WHO guideline on drugs for covid-19. BMJ (online). 2020.

16. Guarascio F. EU urged to review remdesivir supply deal after COVID-19 trial results. 2020 2020-10-16.

17. Fadel R, Morrison AR, Vahia A, et al. Early Short-Course Corticosteroids in Hospitalized Patients With COVID-19. Clinical infectious diseases: an official publication of the Infectious Diseases Society of America. 2020;71(16):2114-20. doi:10.1093/cid/ciaa601.

18. Rezagholizadeh A, Khiali S, Sarbakhsh P, et al. Remdesivir for treatment of COVID-19; an updated systematic review and meta-analysis. Eur J Pharmacol. 2021;897:173926. doi:https://doi.org/10.1016/j.ejphar.2021.173926.

19. Pasquini Z, Montalti R, Temperoni C, et al. Effectiveness of remdesivir in patients with COVID-19 under mechanical ventilation in an Italian ICU. J Antimicrob Chemother. 2020;75(11):3359-65.

20. Fried MW, Crawford JM, Mospan AR, et al. Patient characteristics and outcomes of 11721 patients with coronavirus disease 2019 (COVID-19) hospitalized across the United States. Clin Infect Dis. 2021;72(10):e558-e65.

21. Olender SA, Perez KK, Go AS, et al. Remdesivir for Severe Coronavirus Disease 2019 (COVID-19) Versus a Cohort Receiving Standard of Care. Clin Infect Dis. 2020. doi:10.1093/cid/ciaa1041.

22. Wang Y, Zhang D, Du G, et al. Remdesivir in adults with severe COVID-19: a randomised, double-blind, placebo-controlled, multicentre trial. The Lancet. 2020;395(10236):1569-78. doi:https://doi.org/10.1016/S0140-6736(20)31022-9.

\section{Figures}



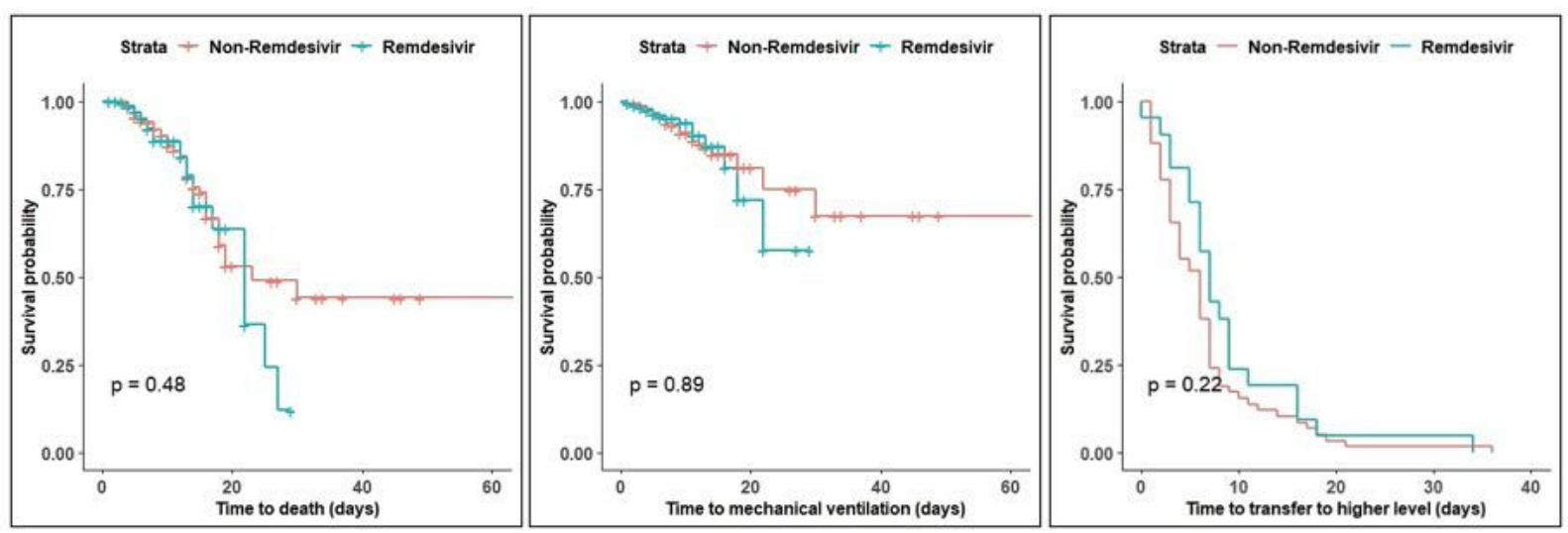

Figure 1

Kaplan Meier curves for mortality, mechanical ventilation, and transfer to higher level.

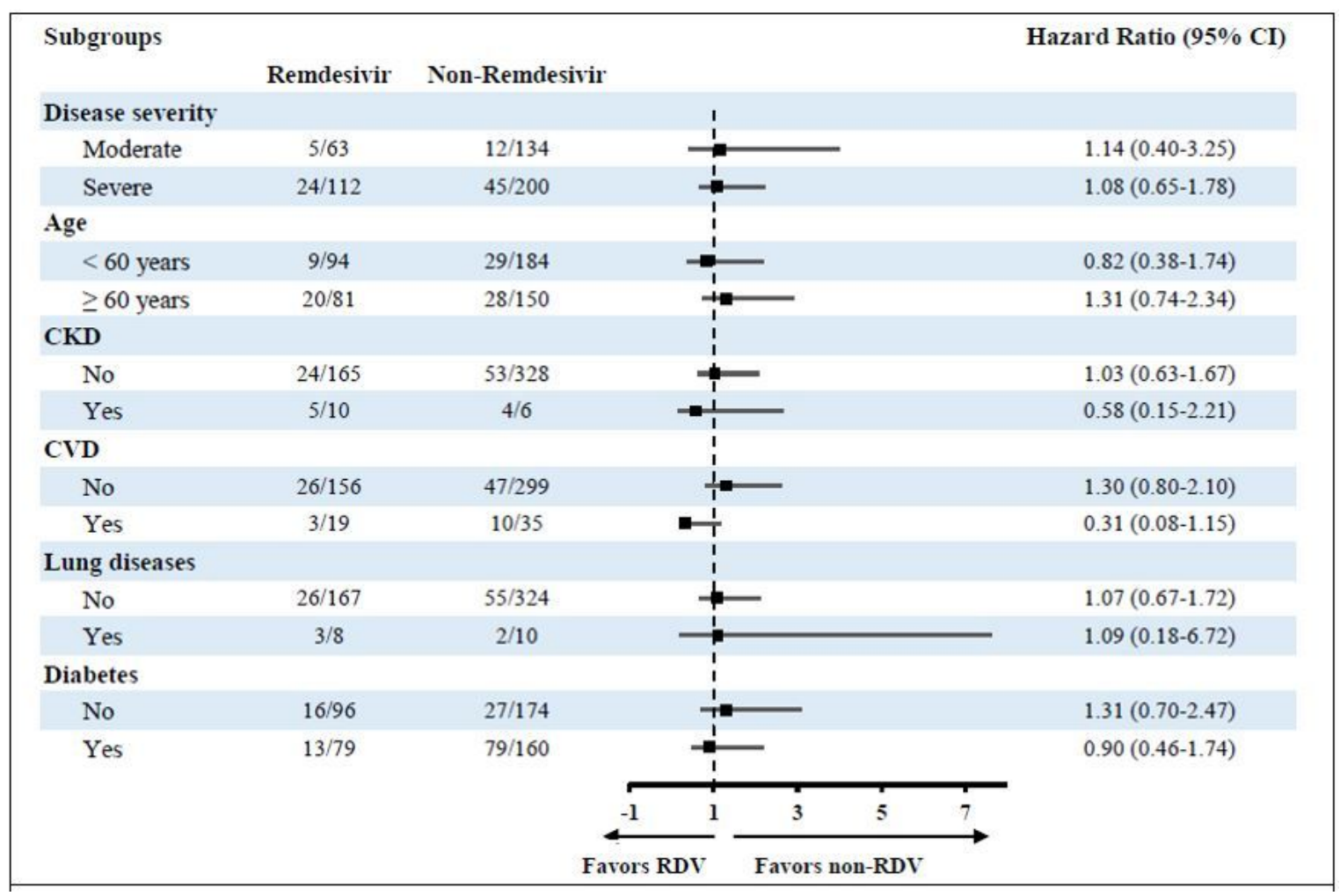

Figure 2

Subgroup analysis of the risk of Mortality in different treatment groups 


\begin{tabular}{|c|c|c|c|c|c|}
\hline \multirow[t]{2}{*}{ Subgroups } & & & & & \multirow[t]{2}{*}{ Hazard Ratio $(95 \% \mathrm{CI})$} \\
\hline & Remdesivir & Non-Remdesivir & & & \\
\hline Disease severity & & & 8 & & \\
\hline Moderate & $6 / 134$ & $3 / 63$ & 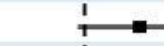 & $\square$ & $3.22(0.75-13.87)$ \\
\hline Severe & $12 / 112$ & $28 / 200$ & $\frac{1}{-1}$ & & $0.72(0.35-1.49)$ \\
\hline \multicolumn{6}{|l|}{ Age } \\
\hline$<60$ years & $6 / 94$ & $19 / 184$ & + & - & $1.65(0.62-4.40)$ \\
\hline$\geq 60$ years & $9 / 81$ & $15 / 150$ & 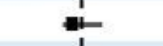 & & $0.61(0.26-1.46)$ \\
\hline \multicolumn{6}{|l|}{ CKD } \\
\hline No & $13 / 165$ & $32 / 328$ & 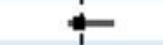 & & $0.90(0.44-1.83)$ \\
\hline Yes & $2 / 10$ & $2 / 6$ & $i$ & - & $1.90(0.24-14.75)$ \\
\hline CVD & & & $i$ & & \\
\hline No & $13 / 156$ & $29 / 299$ & + & & $1.01(0.48-2.10)$ \\
\hline Yes & $2 / 19$ & $5 / 35$ & $\frac{1}{1}$ & & $0.48(0.09-2.56)$ \\
\hline \multicolumn{6}{|l|}{ Lung diseases } \\
\hline No & $14 / 167$ & $32 / 324$ & + & & $1.03(0.52-2.03)$ \\
\hline Yes & $1 / 8$ & $2 / 10$ & $=1$ & & $0.17(0.01-1.93)$ \\
\hline Diabetes & & & $i$ & & \\
\hline No & $9 / 96$ & $17 / 174$ & - & & $0.80(0.34-1.87)$ \\
\hline \multirow[t]{3}{*}{ Yes } & $6 / 79$ & $17 / 160$ & $-\frac{1}{i}$ & & $1.03(0.38-2.82)$ \\
\hline & & & $\begin{array}{rrr}-1 & 1 & 3 \\
\end{array}$ & $\begin{array}{llllll}5 & 7 & 9 & 11 & 13 & 15 \\
\end{array}$ & \\
\hline & & & Favors RDV & Favors non-RDV & \\
\hline
\end{tabular}

Figure 3

Subgroup analysis of the risk of Mechanical ventilation in different treatment groups 